\title{
Myocardial infarction induces cardiac fibroblast transformation within injured and non-injured regions of the mouse heart
}

\author{
Haisam Shah ${ }^{1,2^{*}}$, Alison Hacker ${ }^{1}$, Dylan Langburt ${ }^{1,2}$, Michael Dewar ${ }^{1,2}$, Meghan J. McFadden ${ }^{1}$, \\ Hangjun Zhang ${ }^{1}$, Uros Kuzmanov ${ }^{1,2}$, Yu-Qing Zhou ${ }^{1}$, Bilal Hussain ${ }^{1}$, Fahad Ehsan ${ }^{1,2}$, Boris Hinz ${ }^{3}$, \\ Anthony O. Gramolini ${ }^{1,2^{*}}$, and Scott P. Heximer ${ }^{1,2^{*}}$ \\ ${ }^{1}$ Translational Biology and Engineering Program, Ted Rogers Centre for Heart Research, \\ Toronto, Ontario, Canada M5G 1M1 \\ ${ }^{2}$ Department of Physiology, Faculty of Medicine, University of Toronto, Toronto, Ontario, \\ Canada M5S $1 M 8$ \\ ${ }^{3}$ Laboratory of Tissue Repair and Regeneration, Faculty of Dentistry, University of Toronto, \\ Toronto, Ontario, Canada M5G 1 G6
}

\section{Address Correspondence:}

Dr. Scott P. Heximer, Translational Biology and Engineering Program, Ted Rogers Centre for Heart Research, Department of Physiology, University of Toronto. 661 University Avenue, Toronto, Ontario, Canada M5G 1M1, Phone: 416-978-6048, Email: scott.heximer@utoronto.ca

Dr. Anthony O. Gramolini, Translational Biology and Engineering Program, Ted Rogers Centre for Heart Research, Department of Physiology, University of Toronto. 661 University Avenue, Toronto, Ontario, Canada M5G 1M1, Phone: 416-946-8257, Email: Anthony.gramolini@utoronto.ca

Haisam Shah, Translational Biology and Engineering Program, Ted Rogers Centre for Heart Research, Department of Physiology, University of Toronto. 661 University Avenue, Toronto, Ontario, Canada, M5G 1M1, Phone: +61-416-070-074, Email: haisam.shah@ mail.utoronto.ca 


\section{SUPPORTING INFORMATION - TABLE OF CONTENTS}

Supplemental Figure 1. Raw immunoblots of aSMA and vimentin $3-4$

Supplemental Figure 2. Echocardiography of sham operated and LAD-ligated mice $\quad$ 5-6

Supplemental Figure 3. Histogram of transformed and imputed LFQ values 7

Supplemental Figure 4. Global correlation between individual samples $8-9$

Supplemental Figure 5. Volcano plot comparing sham infarct and sham remote CFs $\quad 10$

Supplemental Figure 6. Volcano plot comparing MI infarct and MI remote CFs 11

Supplemental Figure 7. Raw immunoblots of $\alpha$ SMA, Cthrc1, and vimentin 12

Supplemental Figure 8. Waterfall plot of CF proteins identified in Poulsen et al. $\quad$ 13-14

Supplemental Table 1. List of all unfiltered identifications for the MS experiments

Supplemental Table 2. Filtered list of all 2148 proteins with imputation for the MS experiment

Supplemental Table 3. List of 1025 proteins that were differentially expressed between at least

2 of the experimental groups by multi-comparison ANOVA (FDR $<0.05, \mathrm{~S} 0=1.0$ )

Supplemental Table 4. List of proteins from binary comparison of MI infarct and sham CFs $($ FDR $<0.05, \mathrm{~S} 0=1.0)$

Supplemental Table 5. List of proteins from binary comparison of MI remote and sham CFs $(\mathrm{FDR}<0.05, \mathrm{~S} 0=1.0)$

Supplemental Table 6. List of proteins from binary comparison of MI infarct and MI remote CFs $($ FDR $<0.05, \mathrm{~S} 0=1.0)$

Supplemental Table 7. List of proteins from binary comparison of MI infarct and MI remote CFs with less stringent cut-off (FDR < 0.05)

Supplemental Table 8. GO terms associated with proteins enriched in MI infarct CFs, relative to sham control $(\mathrm{n}=867$ protein)

Supplemental Table 9. GO terms associated with proteins downregulated in MI infarct CFs, relative to sham controls ( $\mathrm{n}=185$ protein)

Supplemental Table 10. GO terms associated with proteins enriched in MI remote CFs, relative to sham control $(\mathrm{n}=505$ protein)

Supplemental Table 11. GO terms associated with proteins downregulated in MI remote CFs, relative to sham control $(\mathrm{n}=74$ protein)

Supplemental Table 12. GO terms associated with proteins differentially expressed between MI infarct versus MI remote CFs ( $\mathrm{n}=285$ protein) 


\section{Supplemental Figure 1}

Days in culture
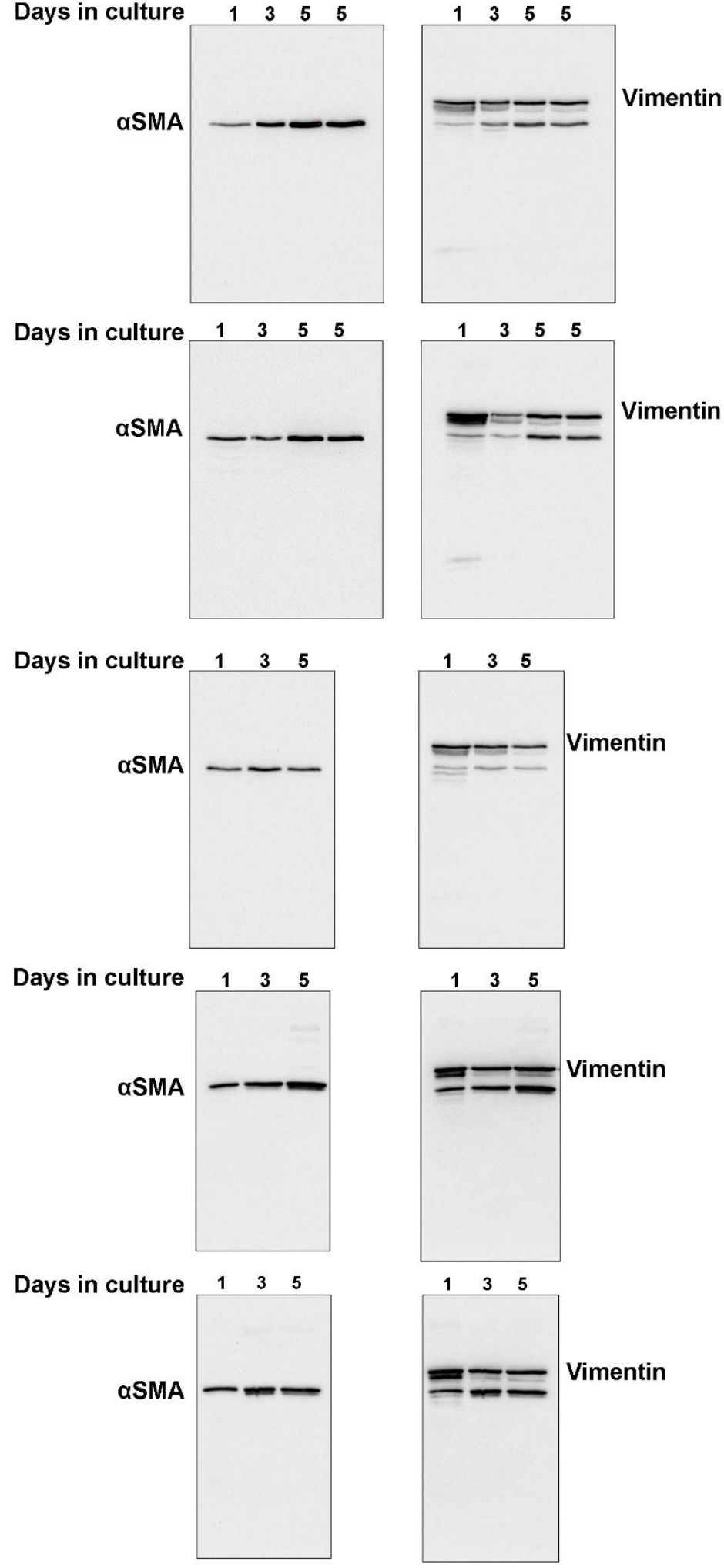
Supplemental Figure 1. Raw immunoblots depicting changes in CF's $\alpha$ SMA and vimentin protein expression over time in culture. All blots were initially probed with anti- $\alpha$ SMA antibody and imaged. The same blots were then incubated with anti-vimentin antibody overnight and imaged again the following day. 
Supplemental Figure 2

A)

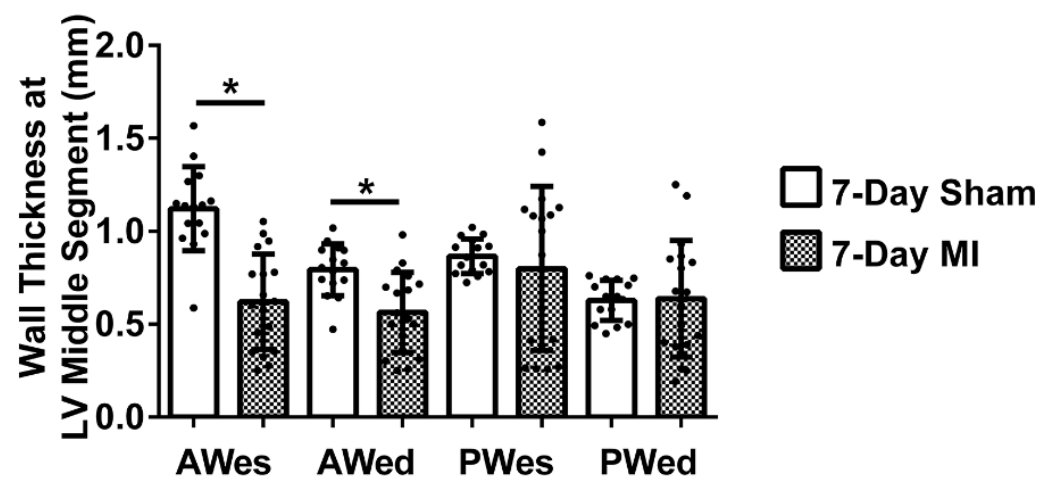

B)
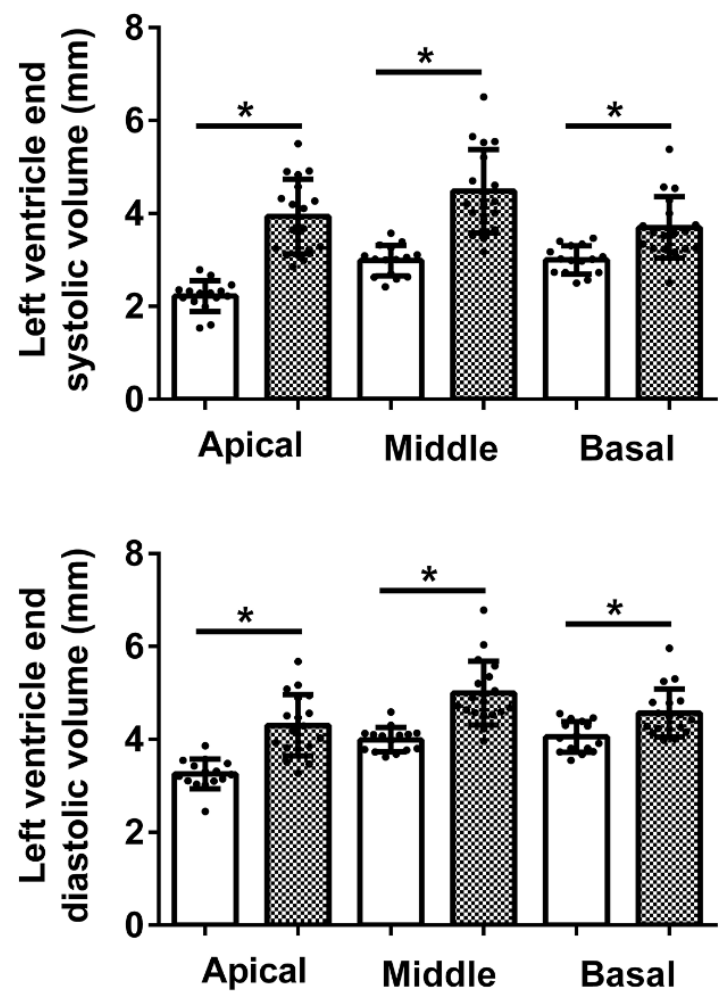

Supplemental Figure 2. Echocardiography showed that MI mice had a thinner LV anterior wall at the middle segment and increased chamber diameter at 7-days following surgery, when compared to sham-operated control mice. Specifically, One-dimension, M-mode echocardiography was performed at the middle segment of the LV 7-days after MI or shamoperation. Tracings were used to assess the thickness of the anterior wall at end systole (AWes), anterior wall at end diastole (AWed), posterior wall at end systole (PWes), and posterior wall at 
end diastole (PWed) [A]. As shown, infarcted hearts had a thinner anterior wall at both end systole and diastole, relative to sham controls. However, no differences were observed in the posterior wall thicknesses. M-mode tracings were also used to assess the LVEDD and LVESD [B]. Our data showed that LV chamber diameter at both end systole and diastole were significantly elevated at the apical, middle, and basal segments of MI mice, relative to sham controls. Significant $\left(^{*}\right)$ at $\mathrm{p}$ $<0.05$ using unpaired, two-tailed, student's t-test comparing sham and MI mice. Graph depicts mean \pm standard deviation. 
Supplemental Figure 3
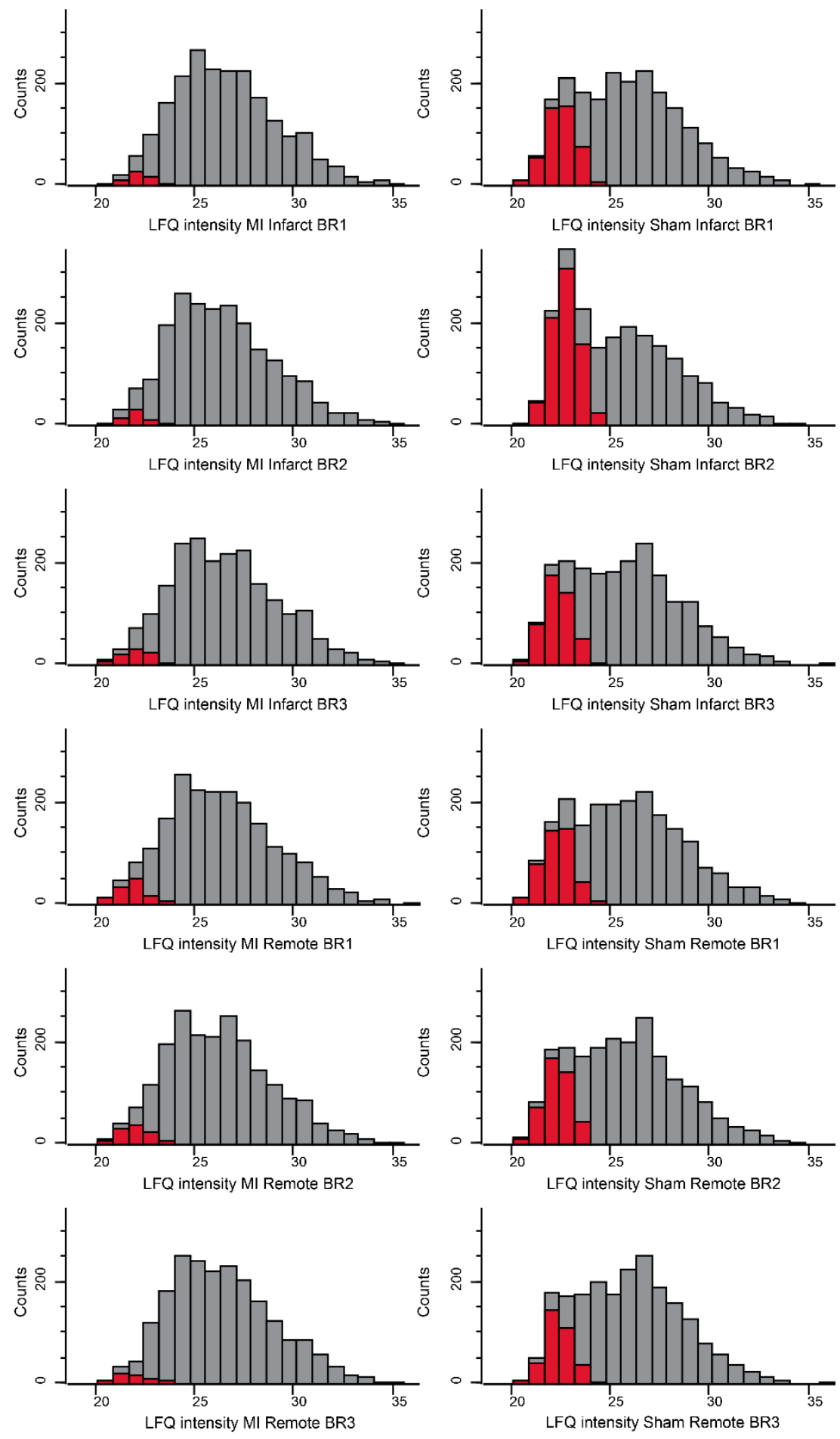

Supplemental Figure 3. Histograms of transformed $\log _{2}(x)$ and imputed LFQ values (in red) for each biological replicate. 


\section{Supplemental Figure 4}
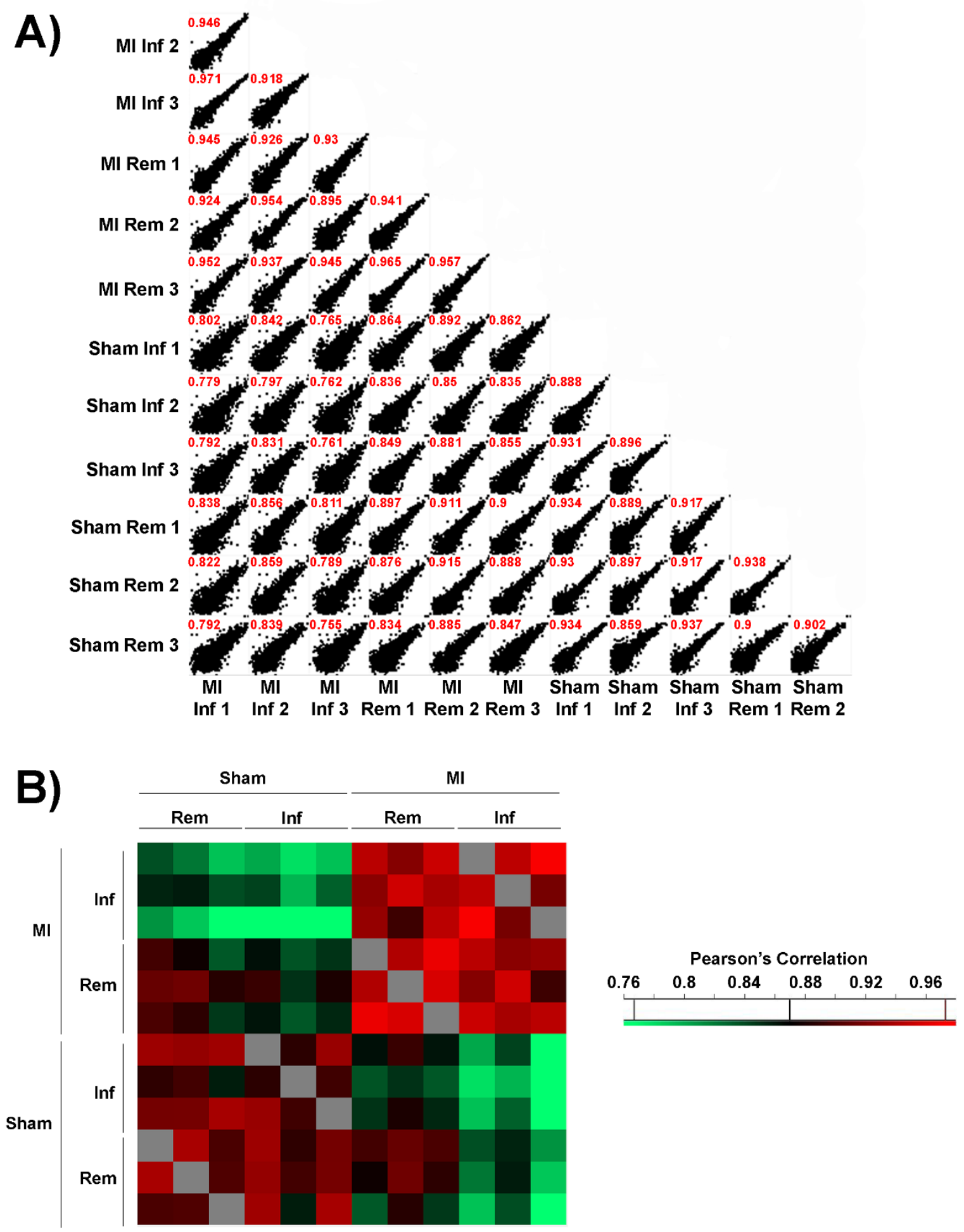


\section{Supplemental Figure 4. Global correlation of transformed $\log _{2}(x)$ and imputed LFQ values}

between individual samples. Pearson correlation coefficient is inset in individual plots $[\mathrm{A}]$. We also generated a heatmap depicting Pearson's correlation values from all possible comparisons (including the individual biological replicates) [B]. The scale depicts the Pearson's correlation ranging from 0.76 (light green) to 0.98 (bright red), which corresponds to relatively weak and strong correlation, respectively. We observed the strongest correlation when comparing MI to other MI samples, or sham to other sham samples. In contrast, the weakest correlations were observed when comparing sham samples to MI infarct (inf) and to a lesser extent when comparing sham to MI remote (rem) samples. 


\section{Supplemental Figure 5}

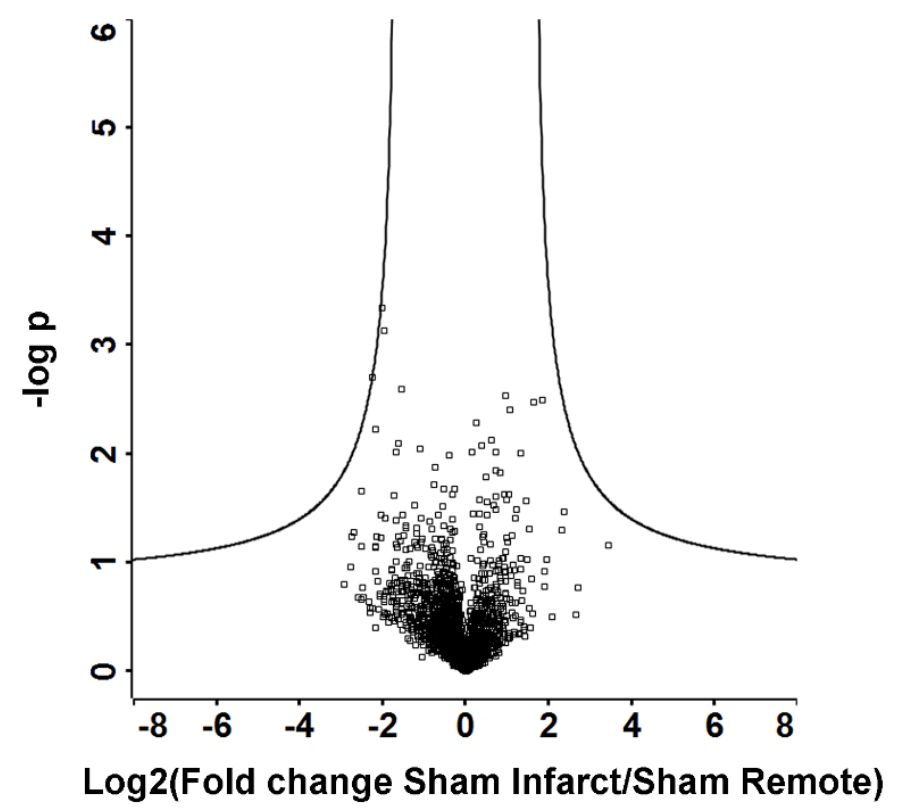

\section{Supplemental Figure 5. Volcano plot comparing sham infarct and sham remote CF samples.}

The plot shows the logarithm (base 2) of the fold change between the mean LFQ values of sham infarct versus sham remote samples on the x-axis and the negative logarithm (base 10) of the pvalue on the $y$-axis. The curved solid black lines are known as the 'line of significance', which are determined based on a permutation-based FDR cut-off of $<0.05$ and a S0 $=1.0$. We found no differentially expressed proteins between CFs from the two regions of the heart. 


\section{Supplementary Figure 6}

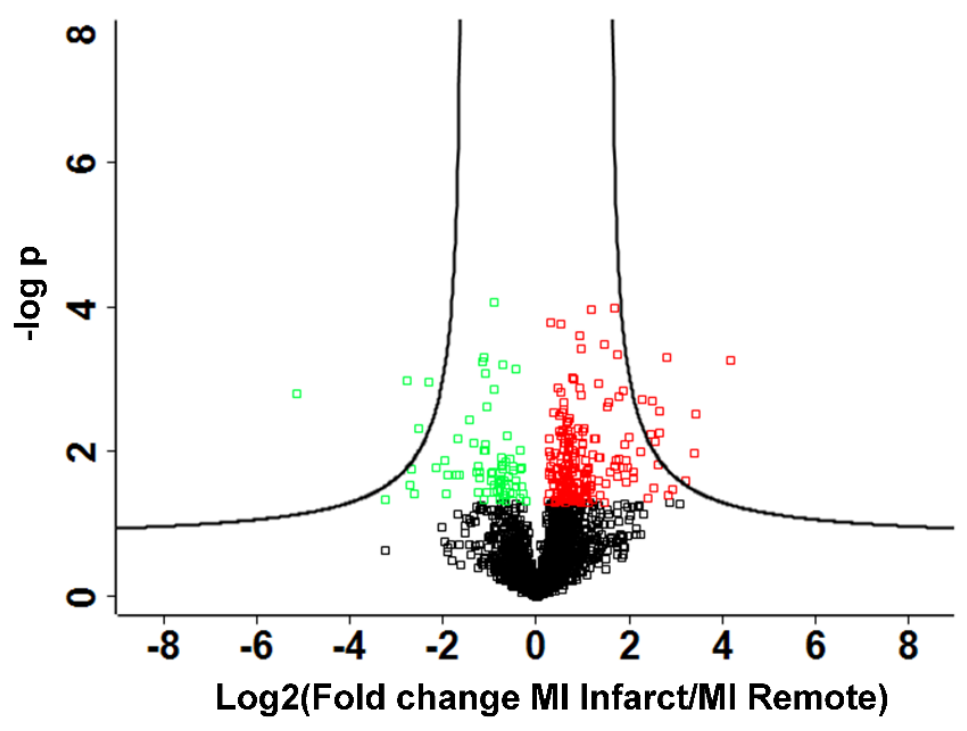

Supplemental Figure 6. Volcano plot comparing MI infarct and MI remote samples. The plot shows the logarithm (base 2) of the fold change between the mean LFQ values of MI infarct versus MI remote samples on the $\mathrm{x}$-axis and the negative logarithm (base 10) of the p-value on the $\mathrm{y}$-axis. The curved solid black lines are known as the 'line of significance', which are determined based on a permutation-based FDR cut-off of $<0.05$ and a $\mathrm{S} 0=1.0$. There were 15 proteins that were significantly altered using this criterion. The red and green squares indicate proteins that were significantly upregulated and downregulated, respectively in the MI infarct samples compared to MI remote based on a FDR $<0.05$. There were 285 differentially altered proteins identified using this less stringent criterion. 


\section{Supplemental Figure 7}
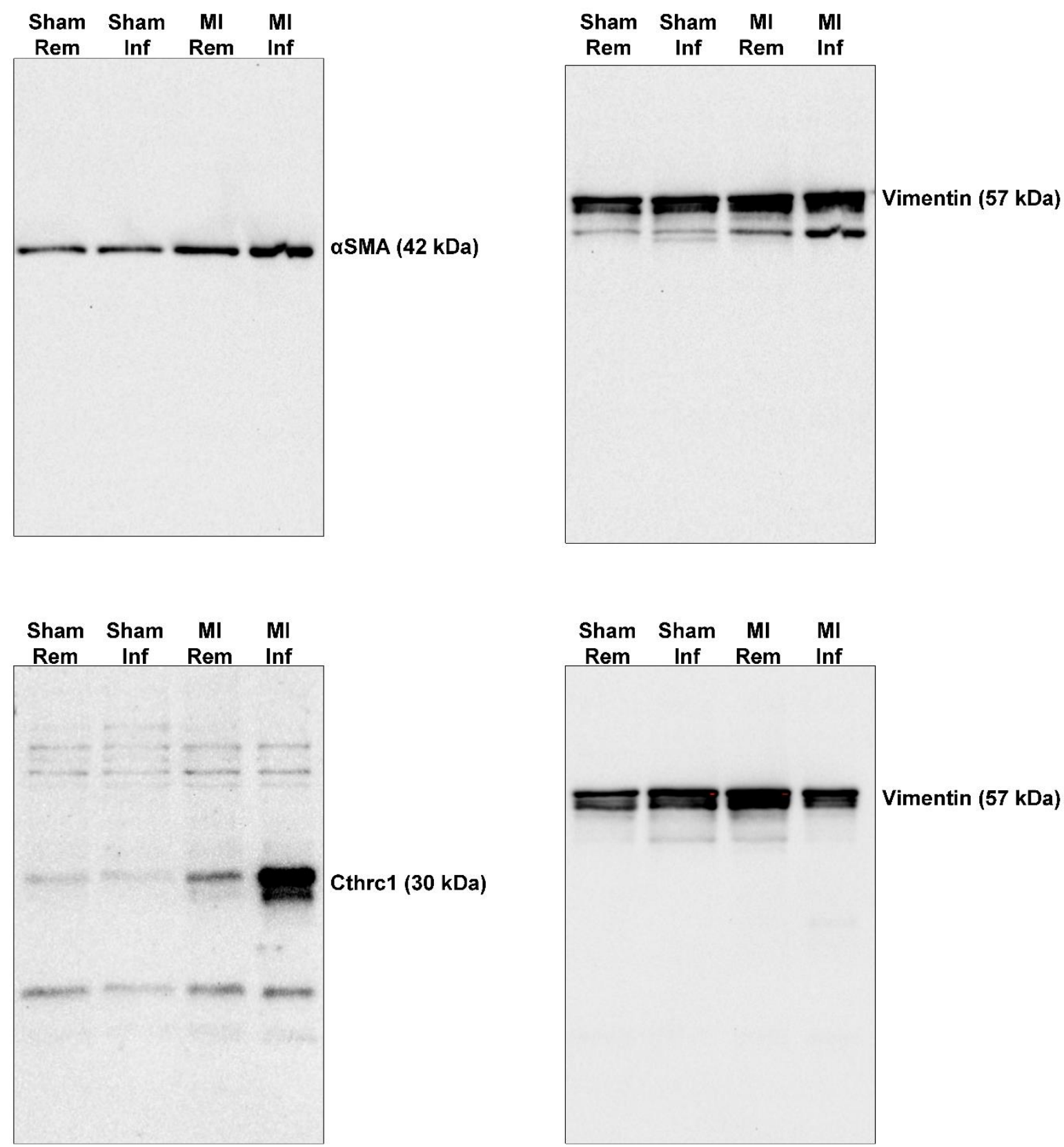

Supplemental Figure 7. Raw immunoblots depicting changes in CF's aSMA and Cthrc1 protein expression in remote and infarct regions of sham or MI operated hearts. Blots were initially probed with anti- $\alpha$ SMA or anti-Cthrcl antibody and imaged. The same blots were then incubated with anti-vimentin antibody overnight and imaged again the following day. 


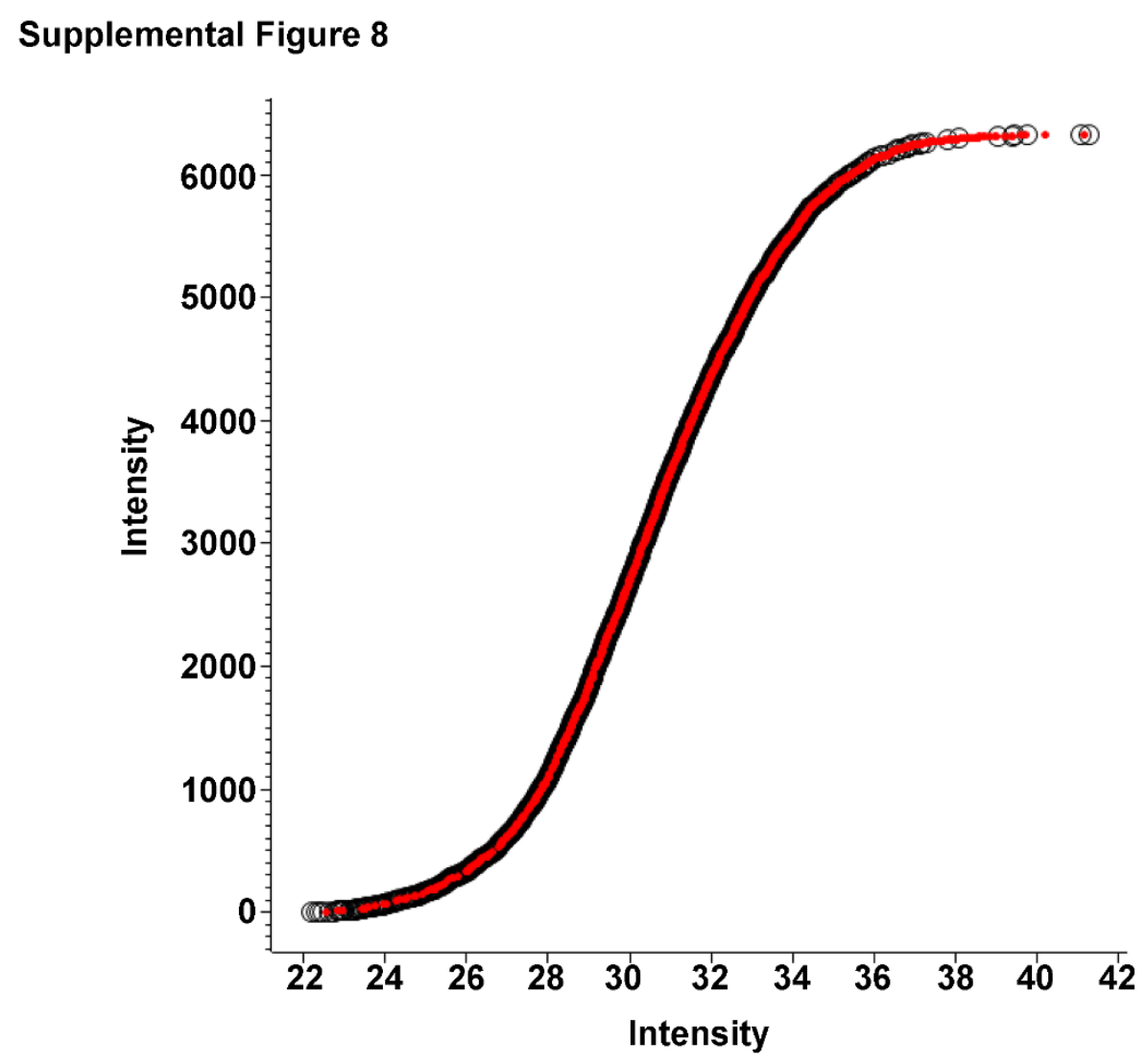

Poulsen et al. Shah et al.

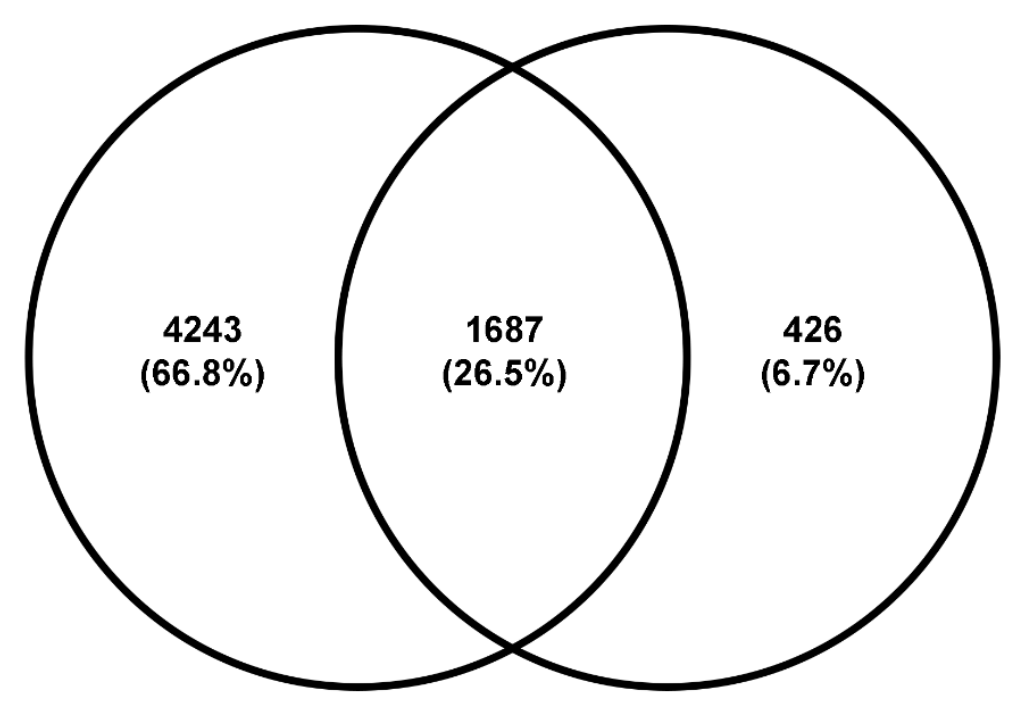

Supplemental Figure 8. Waterfall plot of cardiac fibroblast proteins identified in Poulsen $e t$ $\boldsymbol{a l}$. The $\log 2$ transformed total extracted ion intensities plotted on the x-axis and protein rank (based 
on intensity) plotted on the y-axis. Commonly identified fibroblast proteins from our experiments are detonated as smaller red filled circles and proteins only identified in Poulsen et al. are denoted as large open circles. We also generated a Venn diagram to demonstrate that a large proportion of proteins identified in our study were also identified by Poulsen et al. 